\title{
Analysis of the clinical risk factors of re-fracture after osteoporotic fracture
}

\section{Lin Wang ( $053113653 @ q q . c o m$ )}

The Yijishan Hospital of Wannan Medical College

\section{Guo-Zheng Ding}

Wannan Medical College

Min Yang

Wannan Medical College

Research article

Keywords: osteoporosis, refracture, risk factors

Posted Date: March 30th, 2020

DOI: https://doi.org/10.21203/rs.3.rs-19844/v1

License: (1) This work is licensed under a Creative Commons Attribution 4.0 International License.

Read Full License 


\section{Abstract}

Background To investigate the clinical risk factors of patients with osteoporotic fracture and re-fracture.

Methods A total of 96 patients with osteoporotic fractures and re-fractures who were treated in the relevant department of our hospital from January 2017 to December 2018 were selected as the research objects. By comparing the basic clinical situation of these two groups Analysis to explore the main risk factors after refracture.

Results Through statistical analysis of clinical data, it was found that gender, age, the time from lying down to standing, and the bone mineral density $T$ value were all clinical risk factors for refracture. Women who lie prolonged to stand have a higher risk of re-fracture.

Conclusions The risk factors of re-fracture after treatment in elderly patients with osteoporotic fractures are many and complex. Early investigation and targeted preventive measures can be of great significance to reduce the incidence of re-fractures.

\section{Background}

Osteoporosis is a disease characterized by low bone mass, destruction of bone microstructure, increased bone fragility, decreased bone strength and ease of fracturing. The disease has become a leading cause of morbidity and mortality among the elderly [1]. The prevalence of osteoporosis ranges between 13 to $18 \%$ among women aged above 50 years and 3 to $6 \%$ in men. The bone mass of women and men over 50 years old in Europe. The prevalence of osteoporosis was $21 \%$ and $6 \%$, respectively. The prevalence of osteoporosis in women in the United States and Europe is about three times that of men. In China, the prevalence of osteoporosis based on the bone mineral density of the vertebral body and femoral neck was $20.7 \%$ in women and $14.4 \%$ in men. Compared with that of the United States and Europe, the prevalence of osteoporosis among men in China is relatively higher. With the rise of China's aging population and life expectancy, the number of osteoporosis patients will continue to increase. There are approximately 69.44 million people aged over 50 years that are living with osteoporosis in China. It is estimated that the number of osteoporosis patients will increase from the current 286.6 million to 533.3 million by $2050[4,5]$.

The onset of osteoporosis is often insidious with no obvious symptoms. When the disease progresses to a symptomatic phase, its main clinical manifestations include the presence of pain, a hunchback, and height reduction. Severe cases of osteoporosis can lead to fractures. An osteoporotic fracture can occur during the performance of activities daily living or as a result of minor trauma. It commonly occurs in the vertebral column, hips, distal radius and on the proximal shaft of the humerus. Research has shown that about $50 \%$ of women and $22 \%$ of men above the age of 50 years will suffer from an osteoporosis fracture [6, 7]. In 2010, the total prevalence rate of osteoporosis fracture in people over 50 years old in China was $26.6 \%$. The risk of recurrent or second fractures among patients with osteoporotic fractures is quite high (Klotzbuecher et al. year). The meta-analysis of 49 studies on the risk of re-fracture after 
osteoporotic fractures from different countries and regions revealed that the risk in these patients was double that observed in patients with first-time fractures $(95 \% \mathrm{Cl}=1.7-2.4)$ [8]. Kanis et al. In addition, the results from a recent meta-analysis (11 studies) also showed that recurring fractures were 1.86 times more likely to happen in osteoporotic patients compared to that of patients without a history of fractures in Canada, Europe, and other countries $(95 \% \mathrm{Cl}=1.75-1.98)[9,10]$. Furthermore, the predisposition to refracturing varies from site to site. Some studies have shown that the risk of a second hip fracture is 2 to 2.5 times likely to happen while that of a vertebral fracture and that of a distal radius fracture are 5 and 5.67 times likely to happen, respectively $[11,12]$.

Osteoporotic fractures, especially the hip fractures, are often brittle and complete leading to significant residual disability, mental health problems as well as increased mortality. Hip fractures are the most serious consequence of osteoporosis and account for hospitalizations in $18.2 \%$ of osteoporotic fracture patients. The mortality rate during the first year after hip fracture ranges from 20 to $25 \%$ (). More than half of the survivors experience different degrees of disability, activity limitations and reduced quality of life. Due to poor bone quality (low bone density and strength, increased bone fragility), dwindling calcium and vitamin $\mathrm{D}$ levels and poor vision, the elderly are prone to falls that predispose them to fractures and associated mortality.

In China, clinical experts and researchers have previously studied the incidence and risk factors of refracturing in patients with osteoporotic fractures. The fracture sites identified by domestic research include the hip, vertebral bodies, the radius and the shaft of the humerus. Dependent on the different study follow-up times and treatment regimens, the risk of re-fracture ranged between $2.7 \%-15.9 \%(0$. The average time interval between the first fracture and the second fracture was about 1 year. To identify the risk factors of patients with recurrent fractures, most studies used descriptive statistics to analyze the patients' characteristics, while some used logistic multiple regression model or Cox's proportional hazards regression model. Among them, the multiple regression analysis results of Ruan et al., (year) showed that being female, old age, a history of previous spine or hip fracture, time from lying to standing greater than $20 \mathrm{~s}$ and a (high/low?) BMD value were the identified risk factors associated with second fractures in patients with osteoporosis; while Zhang Yonggui's research results. Zhu Xiaodong's study found that high body mass index, depression and poor blood glucose control were also risk factors of a second fracture (). However, the above studies are based on small samples drawn from a single hospital, and the patients included had three fractured sites (hip, vertebral body, and arm). The studies also used limited and different variables related to patients with osteoporosis and their likelihood of a second fracture. Therefore, it is crucial to study this phenomenon further using a comprehensive evaluation of many variables. In addition, there is a dearth of information on medical resources usage and direct costing following osteoporotic fracture in China. The time-lapse between fractures has also not been well covered in the literature. Therefore, this article analyzes the clinical data of 96 patients with osteoporotic fractures to summarize the risk factors associated with osteoporotic fracture recurrence to generate the evidence required for effectively controlling their occurrence. 


\section{Methods}

\subsection{General setting}

96 patients with osteoporotic fractures (first or recurrent) who were admitted into our hospital from May 2017 to April 2018 were selected as the study subjects. Those with fractures caused by a serious impact such as road traffic accidents and falls were excluded. Patients with other interfering comorbidities were also excluded in this study.

\subsection{Data collection}

The demographic and clinical treatment data of the two groups were collected and this included sex, age, history, time of illness, bone density T-score value, Charlson Comorbidity Index (CCl) and the interval between standing and lying movements. The data was used to determine the high-risk factors that are of clinical significance.

\subsection{Statistical analysis:}

SPSS 16.0 statistical software () was used to analyze the above data. The measurement data was expressed as $\mathrm{X} \pm \mathrm{S}$, and a Chi-squared test was carried to test relationships between categorical variables. The difference was considered statistically significant when $\mathrm{P}<0.05$.

\subsection{Inclusion criteria:}

All patients, whether they just had their first fracture or a re-fracture and are over 55 years old. They should have a diagnosis of osteoporosis; the fracture occurs at any of the following sites: the distal radius, the proximal humerus, the vertebral body, the proximal femur and the acetabulum of the hip bone; the fracture causes are falling injuries. Exclusion criteria: if the fracture phenomenon was caused by severe violence, such as traffic accidents; patients who have bone metabolism-related diseases; patients receiving radiotherapy and chemotherapy; patients taking or who have taken anticoagulants and other fracture-related drugs and food.

\section{Results}

\subsection{The demographic characteristics of osteoporosis patients}

The demographic characteristics of osteoporosis patients in this study are shown in Table 1. There were 48 patients with one-time osteoporotic fractures (11 males, average age of $73.1 \pm 6.35$ and 37 females with an average age of $72.5 \pm 6.18$ ). There also 48 patients with recurrent fractures (18 males had second after osteoporosis with mean age 74. $1 \pm 4.62$, and 30 females, mean age was $75.13 \pm 5.08$ ). In the group, fifteen patients were aged between 60 and 70, 33 were over 70 while in the group 12 patients aged 60 to70 and 36 were over 70 years old. The analysis results of the above data after independent sample ttest are shown in Table 1, 2 and Figure1, 2, 3. 


\subsection{Fracture site distribution}

The distribution of fracture sites among patients with first-time osteoporotic fractures and those with second osteoporotic fractures are shown in table 3 and figure 4 . Among the patients with first-time osteoporotic fractures, 9 cases were humeral shaft fractures (18.8\%), 13 cases were femoral fracture $(27.1 \%) 19$ cases of vertebral fracture (39.6\%), and 7 cases had radial fractures (14.5\%). In patients with recurring fractures, there were 8 cases of humeral shaft fracture accounting for $16.7 \%, 12$ cases of femoral fractures $(25 \%), 17$ cases of vertebral fractures (35.4\%), and 11 cases of radius fractures (22.9 $\%)$. The vertebral body fractures were the most frequent injury reported in both groups of patients followed by femoral (Figure 4) and radial fractures (Figure 5).

2.3 Analysis of clinical risk factors of re-fracturing in patients with osteoporosis

In table 4, it can be seen that T-score value of BMD in patients with osteoporotic fractures was $-3.31 \pm$ 0.23 , against- $3.84 \pm 0.16$ of patients with recurrent fractures ( $p$-value $<0.05, \chi^{2}$ test). As shown by the Clarlson Comorbidity Indices, the patients with more than 4 scores. Most of the patients were divided into 0 and 1 points and the patients with osteopine fracture and osteopine re fracture were divided into two groups. The data were statistically significant $(P>0.05)$. The statistics of the interval between lying and standing are as follows: In the first fracture group, 30 patients distributed in $15-20$ s accounted for $62.5 \%$, and 18 patients $\geq 20$ s accounted for $1837.5 \%$; 15 patients distributed in $15-20$ s accounted for $31.3 \%$ of the patients, and the patients $\geq 20$ s 11 cases accounted for $68.7 \%$; statistical SPSS analysis p-value > 0.05 did not have statistical significance

\section{Discussion}

The statistical analysis showed that the majority of the patients who qualified for inclusion in this study were over 70 years old, and most were female. The number of female patients was nearly twice that of the male patients, which is consistent with the domestic research results on the prevalence of osteoporosis (). In the United States in 2004 and Europe in 2010, women were about three times more likely to suffer from osteoporosis than men. Compared with the United States and Europe, the proportion of male patients in China is higher. The possible reason could be Chinese men's habits such as (excessive) drinking and smoking as well as high pressure might be causing the increased risk of fracture, but the specific reason needs to be further clarified [13]. The common downstream complications arising from these habits that predispose to fractures in osteoporosis patients include hypertension, cerebrovascular disease, diabetes, chronic lung disease, chronic heart disease, and peripheral vascular disease.

Among the first time osteoporotic fracture patients included in this study, the majority of them had vertebral and femoral fractures. The patients with osteoporotic fractures for the second time also presented with vertebral, femoral and radial fractures, in that order of decreasing frequency. The main fracture sites were concentrated in the femur or vertebral bodies. These sites not only affects the but also affects the overall balance ability of patients. The decreased ability to maintain balance makes it easy for 
the patients to fall again, resulting in a second fracture. This result is consistent with the international "prevention and treatment of bone". According to the clinical practice guide of osteoporosis (), the common fracture sites are the spine, hip, distal radius, and proximal humerus. In this study, we found that the mean BMD T-score of patients with first-time osteoporotic fractures was significantly lower than that of patients with second osteoporotic fractures. Patients with osteoporosis fracture. In the 65 year old group, for every 1 SD decrease in BMD, osteoporotic bone was found in men. The risk of fracture increased 1.41 times (1.33-1.51) and 1.38 times (1.28-1.41) in women. Two groups of patients' CCI scores were comparable, with more than three-quarters of the patients having 0, 1 and 2 scores. According to the experimental results, 30 patients (62.5\%) in the first-time fracture group spent $15-20 \mathrm{~s}$ while 18 patients spent more than $20 \mathrm{~s}$ to ambulate from lying to standing. 15 patients with osteoporotic repeat fractures also spent 15-20 s while 11 spent more than $20 \mathrm{~s}$. The differences between groups were, however, not statistically significant at $p=0.05$. Although there was a general tendency towards a rise in the number of patients with better outcomes (reduced ambulation time), the small sample could have reduced the statistical power required to capture the significant changes. In healthy patients, the time from lying down to standing is short since the body functions would be normal. However, the time from lying down to standing in weak patients is longer and is related to the reduced physical strength of the patient.

After their first fracture, the patients with osteoporosis still have a high probability of a secondary fracture even if they are treated effectively. The factors that lead to the recurrence of fractures of the patients with osteoporosis are: 1) the continuous decline of the bone density value of the patients leads to the continuous delay of the process of osteogenesis under the patient's periosteum. As a result, the bone trabecula of the patients gradually thins with time. The load-bearing and transfer functions of the bone are also gradually lost, and this seriously affects the rehabilitation process of the patients with osteoporosis fracture. After a fracture, the bone in this area was injured again, which aggravated the damage. Therefore, there is still a great possibility of secondary fractures even after the patient leaves the hospital. (2) Once the elderly osteoporosis patients have fractures, the possibility of full recovery is low. The immune functions of the elderly patients are decreased and, along with decreased general body functions, the rate of healing is severely compromised. The elderly patients also become prone to opportunistic infections, especially the bedridden. These factors predispose elderly patients to secondary fractures. (3) Following fractures, the elderly osteoporosis patients not only have serious physiological pain, but they also suffer from psychological trauma. This predisposes them to avoidance behaviors that exacerbate their anxieties. These patients become extremely afraid of walking normally, thereby increasing the risk for and incidence of secondary fractures $[14,15]$.

There are many other factors other than age and sex, such as long transition time from lying to standing position and low BDM T-scores that influence predisposition to secondary fractures and warrant attention. More specific preventive measures and careful explanation of osteoporosis to middle-aged and old people should be carried to reduce the incidence of these debilitating fractures. It is also crucial to strengthen awareness of fracture recurrence among patients with osteoporosis. Clinicians and 
biomedical researchers should continue to study various related risk factors and actively seek to reduce the incidence of fracture recurrence, and improve the quality of life of patients.

\section{Conclusion}

The clinical data and personal information of 48 patients with osteoporosis fracture and 48 patients with re fracture after osteoporosis were analyzed. Through the statistics of personal information of the two groups of patients, it is found that the proportion of female patients in the first place is much larger than that of male patients, and the second age is more than 60 years old, mainly distributed over 70 years old. When identifying the specific location of fracture, there are four kinds of fracture: femoral fracture, humeral fracture, radial fracture and vertebral body fracture. Fracture, through the statistics of the fracture site of patients, we found that the overall frequency of four kinds of fractures is similar. In the patients with osteoporosis fracture and the patients with re fracture after osteoporosis fracture, vertebral fracture has a slightly higher proportion, but the overall difference is not significant, and the analysis found that there is no statistical significance. Through t-test and chi square test of t-value of BMD, Charlson's index of comorbidity and time between lying and standing, we found that the mean value of tvalue of $B M D$ in patients with osteoporosis was significantly different from that in patients with osteoporotic re fracture, and chi square test found that there was statistical significance $(P<0.05)$, and the time between lying and standing in patients with osteoporotic re fracture was significantly different $(P$ $<0.05$ ) It was significantly longer than that of osteoporotic fracture patients.

\section{Declarations}

Ethics approval and consent to participate: The consent I obtained from study participants was verbal区 which was approved by the Yijishan ethics committee.

Consent to publish: Not applicable

Availability of data and materials $\square$ The data that support the finding of this study are available from The Orthopaedics Center of Yijishan Hospital but restrictions apply to these data,which were used under licence for current study,and so are not publicly available. Data are however available from authors upon reasonable request and with permission of The Orthopaedics Center of Yijishan Hospital.

Competing Interest: The authors declare that they have no competing interest

Funding: 1"“Peak"cultivation program of scientific research capacity of Yijishan Hospital (GF2019G16), which provide Statistical data collection support

$2 \varangle$ Natural Science Foundation of Anhui Province (1708085QH209) $\varangle$ which provide paper publishing and polishing cost support

Authors Contributions: WL and YM wrote the main manuscript. DGZ prepared all figures and Tables. all Authors read and approved the manuscript 
Acknowledgements: Not applicable

\section{Authors' Information:}

Lin Wang: 53113653@qq.com

Guozheng Ding: wanglin70345@qq.com

Yang Min: yjsyygk@sohu.com

Department of Orthopaedics, The Yijishan Hospital of Wannan Medical College, Western Zheshan Road 2, Wuhu 241002, Anhui, People's Republic of China

\section{References}

[1] Li, H.M., R.J. Zhang, H. Gao, (2018) New vertebral fractures after osteoporotic vertebral compression fracture between balloon kyphoplasty and nonsurgical treatment PRISMA. Medicine (Baltimore), 97(40): p. e12666.

[2] Hernlund, E., A. Svedbom, M. Ivergard, (2013) Osteoporosis in the European Union: medical management, epidemiology and economic burden. A report prepared in collaboration with the International Osteoporosis Foundation (IOF) and the European Federation of Pharmaceutical Industry Associations (EFPIA). Arch Osteoporos, 8: p. 136.

[3] Balasubramanian, A., J. Zhang, L. Chen, (2019) Risk of subsequent fracture after prior fracture among older women. Osteoporos Int, 30(1): p. 79-92.

[4] Yusuf, A.A., T.J. Matlon, A. Grauer, (2016) Utilization of osteoporosis medication after a fragility fracture among elderly Medicare beneficiaries. Arch Osteoporos, 11(1): p. 31.

[5] Jackson, R.D. and W.J. Mysiw, (2014) Insights into the epidemiology of postmenopausal osteoporosis: the Women's Health Initiative. Semin Reprod Med, 32(6): p. 454-62.

[6] Adachi, J.D., G. loannidis, L. Pickard, (2003) The association between osteoporotic fractures and health-related quality of life as measured by the Health Utilities Index in the Canadian Multicentre Osteoporosis Study (CaMos). Osteoporos Int, 14(11): p. 895-904.

[7] Goldshtein, I., A.M. Nguyen, A.E. dePapp, (2018) Epidemiology and correlates of osteoporotic fractures among type 2 diabetic patients. Arch Osteoporos, 13(1): p. 15.

[8] Klotzbuecher, C.M., P.D. Ross, P.B. Landsman, (2000) Patients with prior fractures have an increased risk of future fractures: a summary of the literature and statistical synthesis. J Bone Miner Res, 15(4): p. 721-39. 
[9] Kanis, J.A., O. Johnell, C. De Laet, (2004) A meta-analysis of previous fracture and subsequent fracture risk. Bone, 35(2): p. 375-82.

[10] Desai, R.J., M. Mahesri, Y. Abdia, (2018) Association of Osteoporosis Medication Use After Hip Fracture With Prevention of Subsequent Nonvertebral Fractures: An Instrumental Variable

Analysis. JAMA Netw Open, 1(3): p. e180826.

[11] Salcuni, A.S., V. Morelli, C. Eller Vainicher, (2016) Adrenalectomy reduces the risk of vertebral fractures in patients with monolateral adrenal incidentalomas and subclinical hypercortisolism. Eur $\mathrm{J}$ Endocrinol, 174(3): p. 261-9.

[12] Chen, C.W., T.L. Huang, L.T. Su, (2013) Incidence of subsequent hip fractures is significantly increased within the first month after distal radius fracture in patients older than 60 years. $\mathrm{J}$ Trauma Acute Care Surg, 74(1): p. 317-21.

[13] Li Gengxing and Heng Weixiang,( 2016) clinical risk study of re fracture after osteoporotic fracture, 16(39): p. $71+74$.

[14] Kerschan-Schindl, K., (2016) Prevention and rehabilitation of osteoporosis. Wien Med Wochenschr, 166(1-2): p. 22-7.

[15] Erny, F., A. Auvinet, D. Chu Miow Lin, (2015) Management of osteoporosis in women after forearm fracture: data from a French health insurance database. Joint Bone Spine, 82(1): p. 52-5.

\section{Tables}

Due to technical limitations, the tables are only available as a download in the supplemental files section.

\section{Figures}




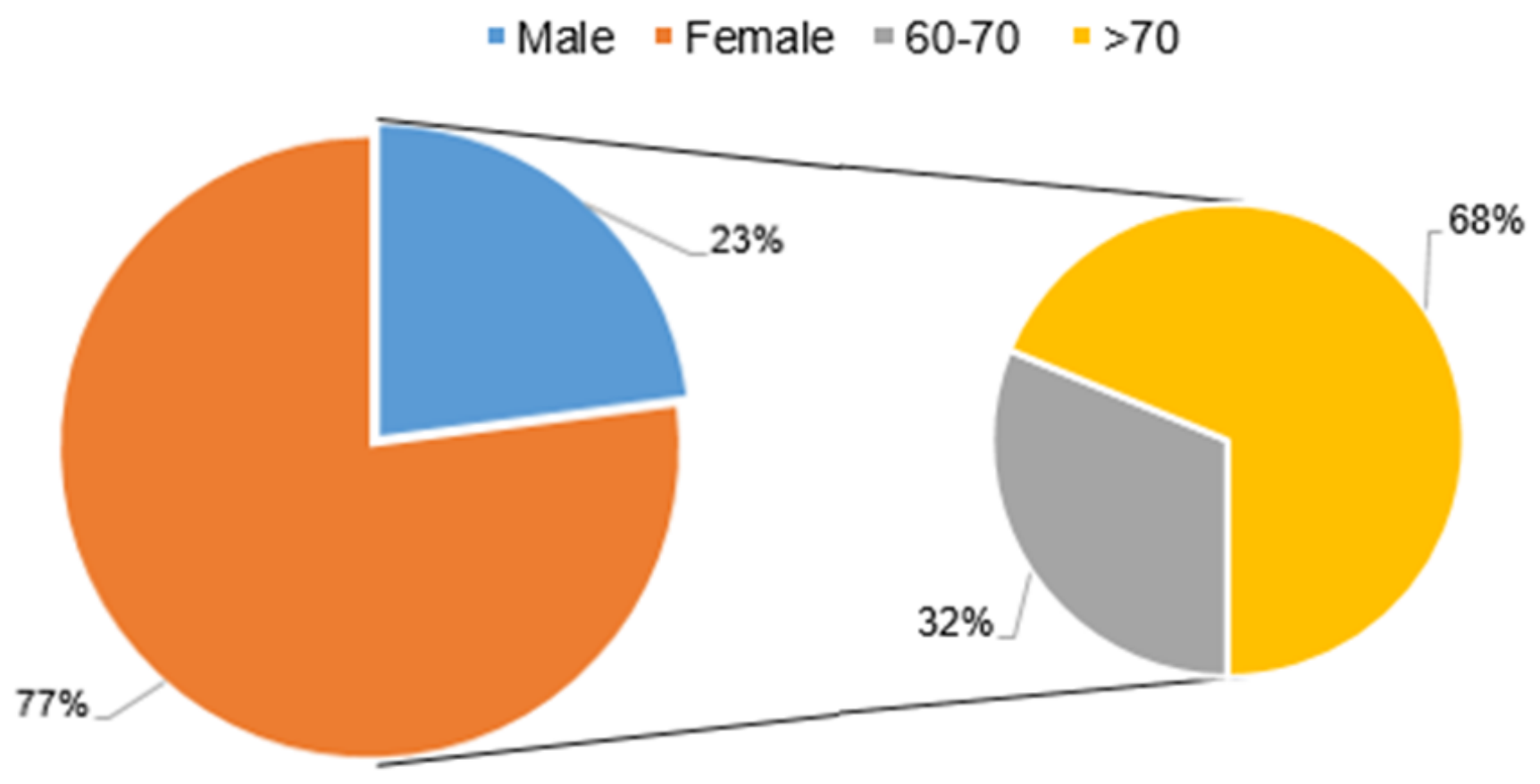

Figure 1

There were 48 patients with osteoporosis fracture, including 11 males which percentage is $23 \%$, 18 females which percentage is $77 \%$. In terms of the two groups, there were 15 patients aged $60-70,33$ patients aged over 70 . The percentage of the patients aged $60-70$ is $32 \%$. The percentage of the patients aged over 70 is $68 \%$ 


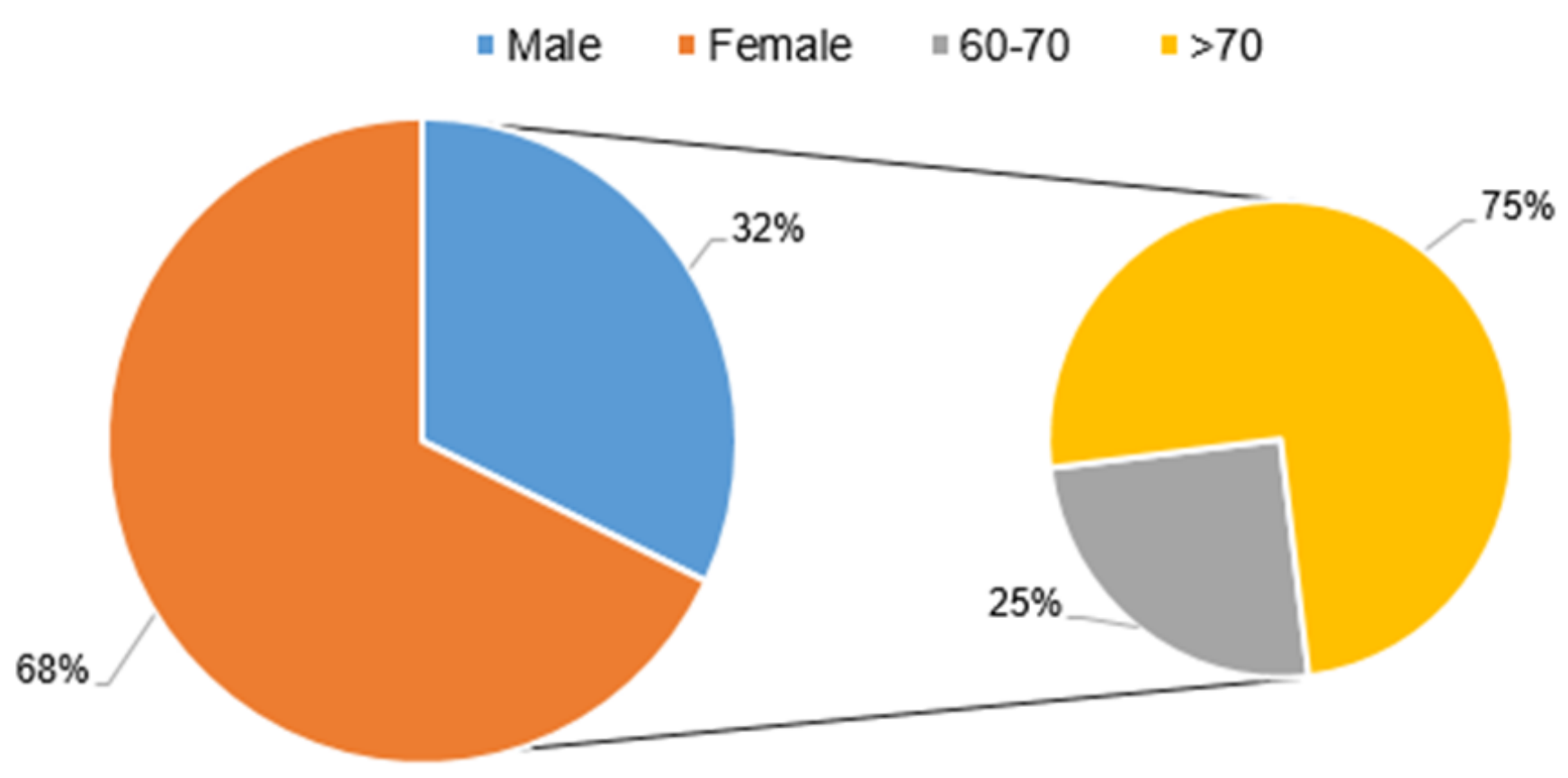

Figure 2

The patients of osteoporotic re-fractures. The percentage of males is $32 \%$, The percentage of females is $68 \%$. The percentage of the patients aged $60-70$ is $25 \%$. The percentage of the patients aged over 70 is $75 \%$ 


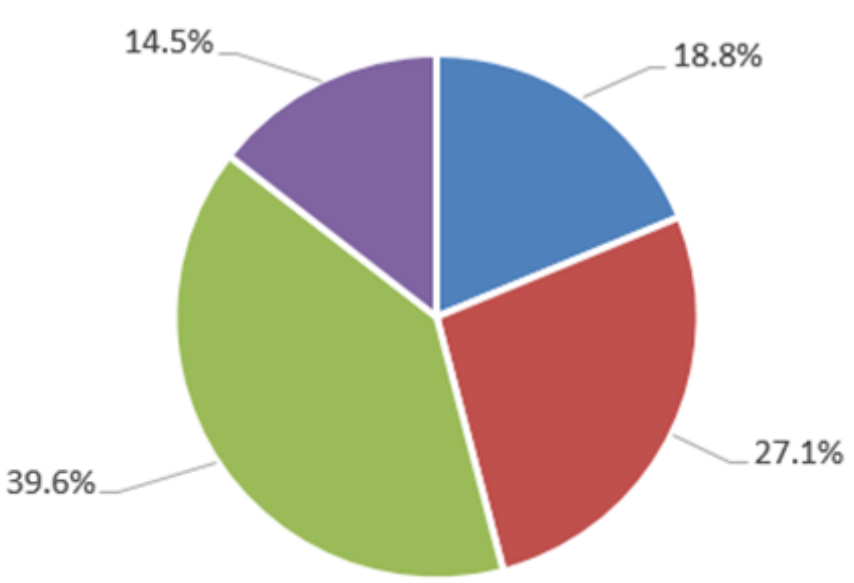

- Humeral fracture = Femoral fracture

- Veterbera fracture = Radius fracture

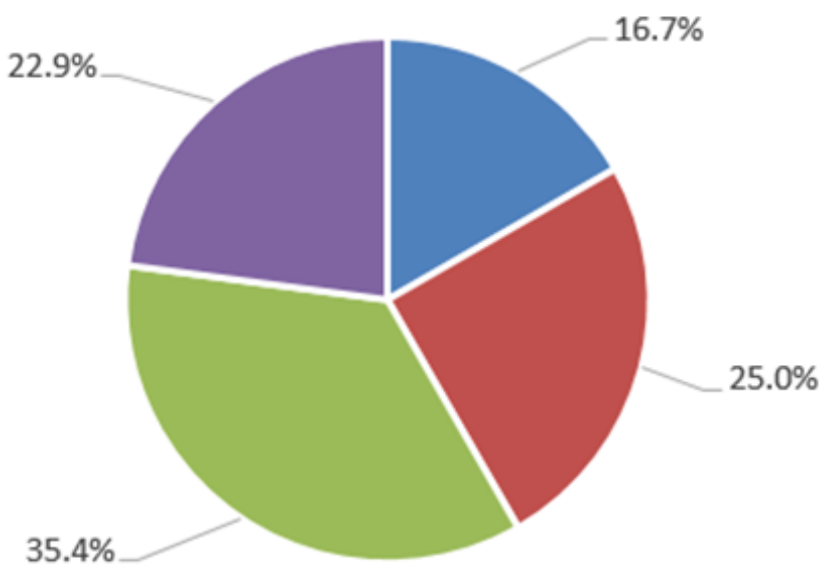

- Humeral fracture $\quad$ - Femoral fracture

- Veterbera fracture $\quad$ - Radius fracture

\section{Figure 3}

Proportion map of fracture sites in patients with osteoporotic fractures and patients with osteoporotic refractures. The percentage of osteoporotic fractures in Humeral fracture, Femoral fracture, Veterbera fracture, Radius fracture is $18.8 \%, 27.1 \%, 39.6 \%, 14.5 \%$. The percentage of osteoporotic re-fractures in Humeral fracture, Femoral fracture, Veterbera fracture, Radius fracture is $16.7 \%, 25.0 \%, 35.4 \%, 22.9 \%$. 


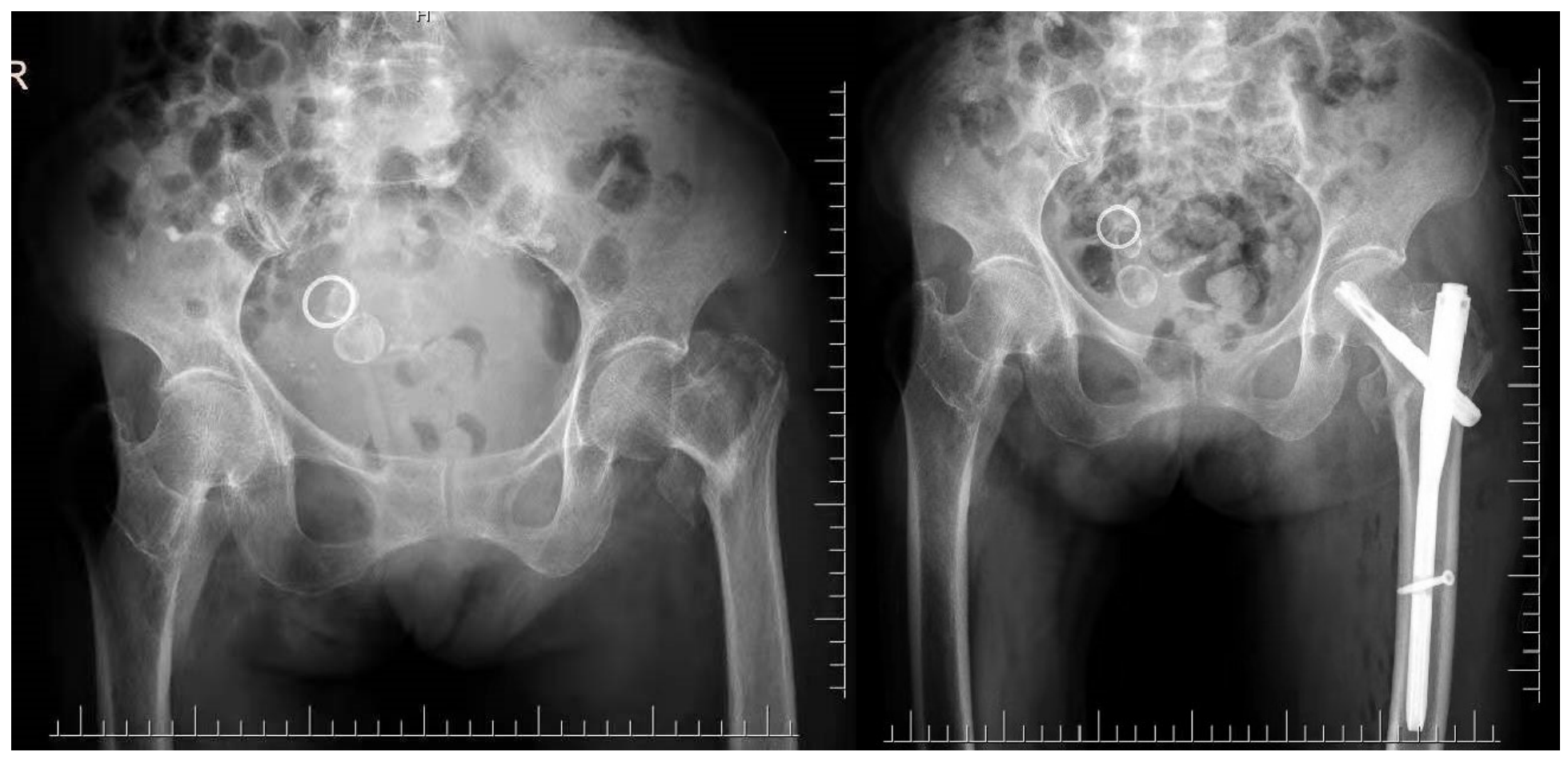

\section{Figure 4}

A patient with a femoral fracture. The patient got femoral fracture with osteoporosis. He got a internal fixation with screw. 

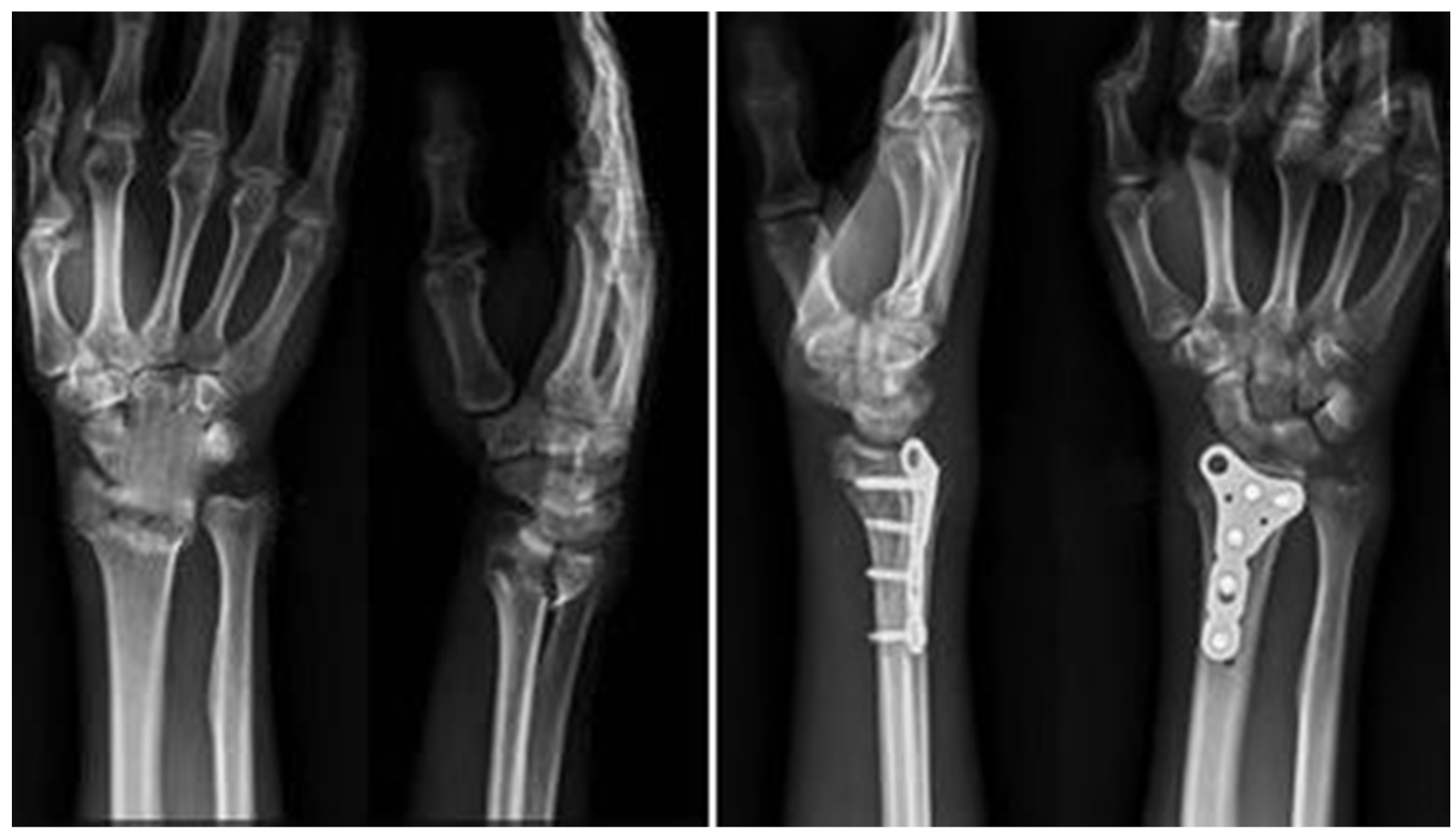

\section{Figure 5}

A patient with a radial fracture. The patient got radius fracture with osteoporosis. He got a internal fixation with plate.

\section{Supplementary Files}

This is a list of supplementary files associated with this preprint. Click to download.

- tables.doc 